\title{
The Static Characteristic Loop and the External Demagnetizing Factor
}

\author{
J. Takacs ${ }^{1 *}$, Gy. Kovacs ${ }^{2}$, L. K. Varga ${ }^{3}$ \\ ${ }^{1}$ Department of Engineering Science, University of Oxford, Oxford, UK; ${ }^{2}$ Department of Materials Physics, Eötvös Loránd Univer- \\ sity, Budapest, Hungary; ${ }^{3}$ Research Institute for Solid State Physics and Optics, Budapest, Hungary. \\ Email: ${ }^{*}$ jenotakacs@aol.com
}

Received June $22^{\text {nd }}, 2012$; revised July $26^{\text {th }}, 2012$; accepted August $29^{\text {th }}, 2012$

\begin{abstract}
In this paper we demonstrate, that shearing is changing only one parameter of the static loop. By using the shearing factor $N_{s}$, linked to the widely used, demagnetization coefficient $N_{D}$, we show the one parameter link between the static un-sheared and that of the sheared saturation loop, obtained by a non-toroidal, open circuit hysteresis measurement. The paper illustrates the simple relation between open circuit loop data and measured real static saturation data. The proposed theory is illustrated by using the hyperbolic model. For experimental illustration, tests results are used, which were carried out on two closed and open toroidal samples, made of NO Fe-Si electrical steel sheet, mimicking the demagnetization effect of the open circuit VSM measurement. These are both theoretical and experimental demonstrations, that shearing only changes the inclination of the static hysteresis loop. These test results, presented here, agree very well with the calculated results, based on the proposed method.
\end{abstract}

Keywords: Magnetism; Hysteresis; Modelling; Shearing

\section{Introduction}

A practical way of characterising a magnetic material is to measure its static hysteresis loop in a closed magnetic circuit. This usually takes form in a toroid or an Epstein square [1], which minimises most of the stray and side effects of other measuring methods. This however is difficult in practice, considering the shapes and sizes of the samples of the magnetic materials in industrial applications. Since the shape of the sample changes its measured magnetic properties, it is desirable to use samples from the final product, which normally come in the shape of a rod or a sheet, for industrial purposes. This however creates some difficulties, with making them into a toroid or Epstein square sample. Most test results therefore are obtained by using VSM (Vibrating-sample Magnetometer) [1-3] method. This method, with excellent repeatability, is now a widely accepted for industrial tests. The data however suffers from the effect of demagnetization, due to the open circuit geometry of the measuring arrangement. The need, to obtain the real, static parameters from measured data of the tested materials, has motivated people to work out various methods to reduce the effects, influencing the measured data [4-7].

At the beginning we have assumed that, only one of

*Corresponding author. the calculated parameters is affected by the external demagnetization. Test results showed, that this assumption was right. It is possible to work out the un-sheared parameters (equivalent to closed circuit toroidal measurements) from the open circuit results of a VSM results. It will be shown, that this procedure requires only change in the effective slope of the loop with no change in the other major parameters of the modelled sample [8].

It was initially assumed that, the error coming from the ever present internal demagnetisation and from other sources is smaller than the [9] (usually at least an order of magnitude smaller) effect of shearing. This is however only conditionally true and all major models (like the Moving Preisach and Dinamic Jiles-Atherton etc.) $[10,11]$ are modified to include, the ever present, internal demagnetization. We also assumed that the effect of shearing depends only on the magnitude of the demagnetization factor and the mechanical treatment not introducing changes in the magnetic properties of the materials. Also assumed, that it is independent of the method of measurement.

Cullity, who studied the effect of shearing on the internal demagnetization in detail, published the relation specifically to the shape and size of the specimen in graphical form [11-15]. In spite of all the assumptions made, the agreement between the theoretical and ex- 
perimental results is remarkable. Further experiments are in progress for further verification this simplified method. Work is also extended to include the so far neglected effects to improve on the accuracy.

\section{Measuring Arrangements}

Preceding the test, the toroidal sample was carefully demagnetized by applying an alternating field of $f=10 \mathrm{~Hz}$ with logarithmically decreasing amplitude in 5000 steps from saturation to zero $[1,2]$. The hysteresis loop was measured with a triangular excitation at a frequency of $f=0.001 \mathrm{~Hz}$ and integrated with a Walker integrator (see Figure 1). The rate of change of the field here is dependent on both the frequency $f$ and field amplitude $H_{m}$ therefore at $H_{m}=20 \mathrm{~A} / \mathrm{m}$, the rate of change, comes to $\mathrm{d} H / \mathrm{d} t=4 f \cdot H_{m}=0.08 \mathrm{~A} / \mathrm{m} \cdot \mathrm{s}$.

The static hystersis loop can only be obtained at low rate of change, particularly when the extremely soft sample has a characteristic square-like hysteresis loop $[1,2]$.

To avoid phase shift between $H$ and $B$, the current measuring resistance $R$ (see Figure 1) has always satisfied the $2 \pi f L<<R$ criterion.

Although the same test was repeated on ultrasoft, Finemet, from nanocrystalline to $\mathrm{Mn}-\mathrm{Zn}$ ferrite, $\mathrm{NO} \mathrm{Fe}-\mathrm{Si}$ and low and high carbon steel cores, due to the limited length of the paper, here only the data of one illustrative experiment are included.

\section{Description of the Model}

The analytical approach is based on the assumption, that in general, particularly in case of soft irons, there are three parallel processes, dominating the overall magnetization process i.e. the reversible and irreversible domain wall movement (DWM), the reversible and irreversible

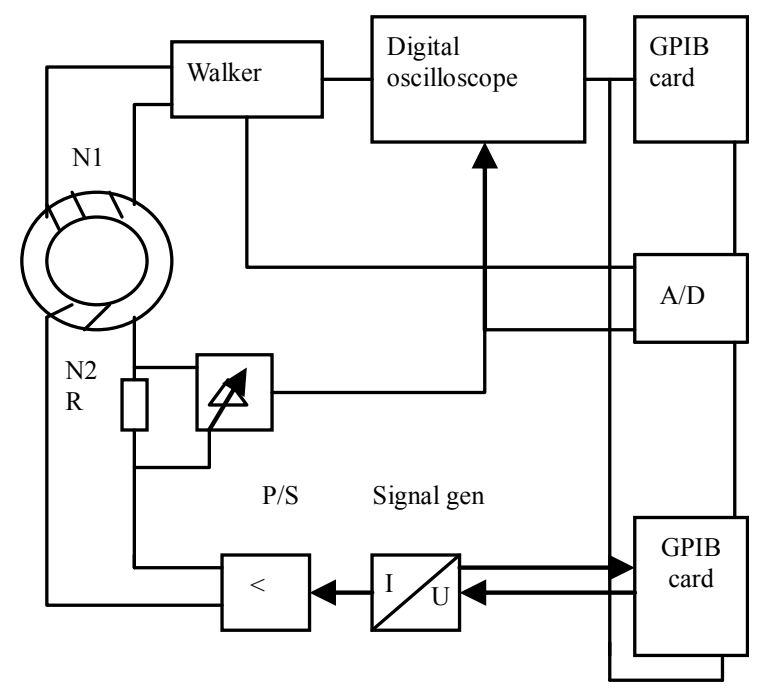

Figure 1. Schematic block diagram of an experimental arrangement for measuring the toroid sample. domain rotation (DR) and the domain wall annihilation and nucleation (DWAN) processes. Although these processes are interlinked, they can be mathematically formulated separately and combined, by using Maxwell's superposition principle. They individually dominate the low, middle and near saturated region of magnetization and all are supposed to have sigmoid shapes. This model is already used in number of applications [15-18] and it is well documented in the literature therefore, here only a brief summary of the relevant formulation will be given in, canonic form $[19,20]$.

The contribution of the individual processes to the combined hysteresis loop can be described by the following normalized mathematical equations.

$$
\begin{aligned}
m_{u} & =\sum_{k=1}^{n}\left(a_{k} f_{u k}+f_{0 k}\right) \\
m_{d} & =\sum_{k=1}^{n}\left(a_{k} f_{d k}-f_{0 k}\right) \\
f_{u k} & =\tan \mathrm{h}\left[\alpha_{k} n_{S}\left(h-h_{c k}\right)\right] \\
f_{d k} & =\tanh \left[\alpha_{k} n_{S}\left(h+h_{c k}\right)\right] \\
f_{0 k} & =\frac{1}{2} \sum_{k=1}^{n} a_{k}\left(f_{u k}-f_{d k}\right) \text { for } h=h_{m}
\end{aligned}
$$

Here $f_{u k}$ and $f_{d k}$ are the normalized ascending and descending magnetization functions respectively, $h$ is the field excitation and $h_{c k}$ is the coercivity of the $k^{\text {th }}$ process. $a_{k}$ is the amplitude of the component processes present, $\alpha_{k}$ is the slope, $n_{S}$ is the shearing factor $\left(n_{S}\right.$ is unity for un-sheared loops, see later) and $f_{0 k}$ is the integration constant [7,21], while $h_{m}$ represents the maximum field excitation. The index $k$ refers to the individual component processes and $n$ is the total number of processes involved. For most of the magnetic materials used in practice, $n$ equals 3 (see beginning of this section).

\section{Experimental Results}

We proposed at the beginning, that the changes in the shape of the hystetesis loop measured on a closed toroid can be described by an appropriate change in the $\alpha_{k} n_{S}$ product (see Equation (2)). Here $\alpha_{k}$ represents the slope of the major loop, measured on a toroid sample (for $n_{S}=$ $\left.1, n_{D}=0\right)$ in the model. For demonstration, we used the measurements on a toroidal sample made of $\mathrm{NO} \mathrm{Fe-Si}$ soft magnetic material. The geometrical details of the toroids used, in the experiment, were: $D_{e x}=25 \mathrm{~mm}, D_{\text {in }}=$ $15 \mathrm{~mm}$ and thickness $\mathrm{d}=0.5 \mathrm{~mm}$ [1].

In the first part of the experiment, the toroid was magnetised to $B_{m}=1.68 \mathrm{~T}$ by applying a linearly variable field of $H_{m}=2750 \mathrm{~A} / \mathrm{m}$ maximum amplitude for measuring the hysteresis curve. Then, two $0.5 \mathrm{~mm}$ air gaps 
were cut in the magnetic circuit at a slow rate and the measurement was repeated. Great care was taken not to introduce additional side effects at cutting. This was for the simulation of an open-loop VSM measurement with the same $N_{D}$ and $N_{S}$. The two hystetesis loops (before and after cutting) are shown in Figure 2. The measured loop, without the gap, was modelled by using Equations (1)-(3). When the iteration (manual or computerised) produced the best fit to the measured curve, the normalized and the equivalent physical values can be easily be read from the two coordinate systems (normalized and measured), as shown in Figure 3.

The iteration yielded the following normalised (lower case letters) and corresponding physical parameters (capital letters) for the best fit:

$$
\begin{aligned}
& a_{1}=1.17, a_{2}=0.14, a_{3}=0.35, \\
& \alpha_{1}=4.5, \alpha_{2}=1.2, \alpha_{3}=0.143, \\
& h_{c 1}=0.115, h_{c 2}=0.45, h_{c 3}=0.575, \\
& h_{m}=7.5 . \\
& \text { Corresponding to: } \\
& A_{1}=1.17 \mathrm{~T}, A_{2}=0.14 \mathrm{~T}, A_{3}=0.35 \mathrm{~T}, \\
& H_{c 1}=44 \mathrm{~A} / \mathrm{m}, H_{c 2}=164.9 \mathrm{~A} / \mathrm{m}, H_{c 3}=210.75 \mathrm{~A} / \mathrm{m} . \\
& H_{m}=2750 \mathrm{~A} / \mathrm{m} .
\end{aligned}
$$

The normalization: $1 b=1 \mathrm{~T}, 1 h=382.6 \mathrm{~A} / \mathrm{m}$.

By altering the shearing coefficient $n_{s}$, in the iteration and using the new values of $\alpha_{1} \cdot n_{S}=0.28, \alpha_{2} \cdot n_{S}=0.073$, and $\alpha_{3} \cdot n_{S}=0.0087$, we obtained the closest fit to the sheared hystetesis loop. The best fit appeared to be, when $n_{S}$ was around 0.061 .

From the geometry of the magnetic circuit, by using the magnetic Ohm's law, $N_{D}$ (see Equation 7 for the relation between $N_{D}$ and $N_{S}$ ) the demagnetization factor, can be calculated by using Equations (4) and (5). We assumed here, that the amplitude of the magnetization vector is the same in the iron as in the air gap. So:

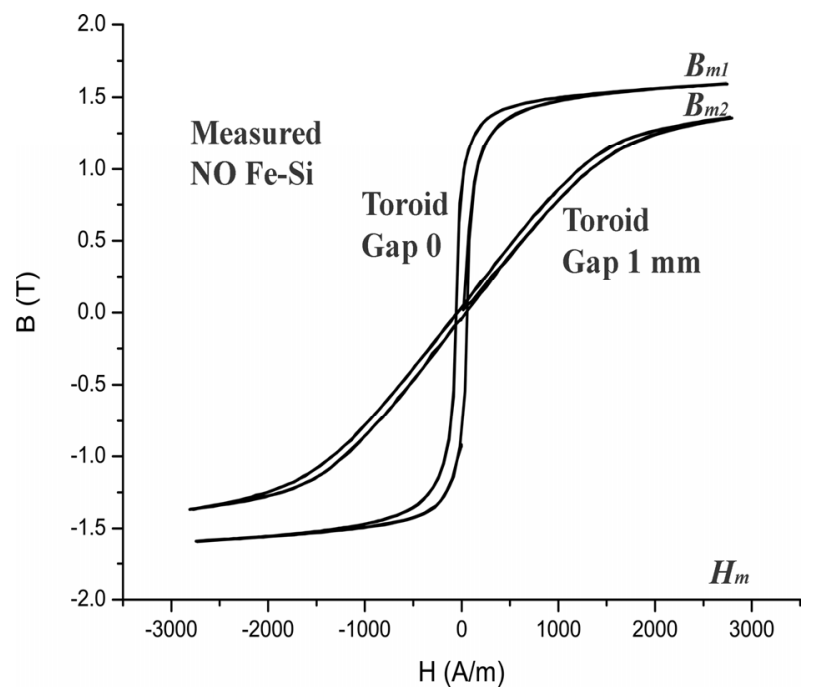

Figure 2. The major hysteresis loops of a NO Fe-Si sample measured on a toroid with and without $1 \mathrm{~mm}$ gap.

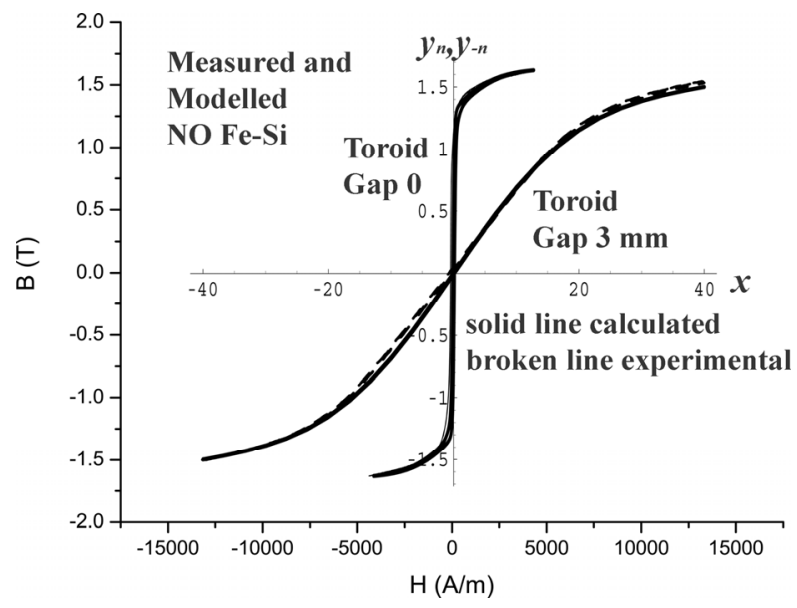

Figure 3. The measured and calculated hysteresis loops of NO Fe-Si material with and without external demagnetization.

$$
B=\frac{\Phi}{A}=\frac{H\left(l_{a}+l_{i}\right)}{\frac{l_{i}}{\mu_{i}}+\frac{l_{a}}{\mu_{a}}}=\frac{H \mu_{i}}{\frac{l_{i}}{l_{a}+l_{i}}+\frac{l_{a}}{l_{a}+l_{i}} \frac{\mu_{i}}{\mu_{a}}}
$$

when $\mu_{i r}$ relative permeability is used and it is assumed that $l_{a} \square \quad l_{i}$ then

$$
B=\frac{H \mu_{i r} \mu_{0}}{1+N_{D} \mu_{i r}}=\mu_{i r} \mu_{0} H_{\text {eff }}
$$

where

$$
H_{\text {eff }}=H N_{S}
$$

is the effective magnetic field excitation.

$$
N_{S}=\frac{1}{1+N_{D} \mu_{i r}}
$$

or in normalised form

$$
n_{S}=\frac{1}{1+n_{D} \mu_{i r}}
$$

Here $l_{i}$ and $l_{a}$ are the mean paths of iron and air respectively, $\mu_{i}$ and $\mu_{a}$ represent the permeability of iron and air (free space) and $A$ is the cross section area of the iron core. It shows that, when $N_{S}$ the shearing coefficient is unity (no shearing), then the $N_{D}$ demagnetization factor is zero. The expression in (9) represents the effective permeability.

$$
\mu_{i e f f}=\frac{\mu_{i r}}{1+N_{D} \mu_{i r}}=\mu_{i r} N_{S}
$$

We must remind the reader of the following: $N_{D}$ is given traditionally in a value with unity dimension, (i.e. when both the $H$ and $B$ measured in $\mathrm{A} / \mathrm{m}$ ). When different unitary system is used then $N_{D}$ has a different physical dimension and must be normalised (see $n_{D}$ as normalised $N_{D}$ ). 
By using the geometrical details of the toroid, given above with $1 \mathrm{~mm}$ effective gap, the physical shearing coefficient numerical value will come to

$$
N_{S}=\frac{1}{1+N_{D} \mu_{i r}} \approx \frac{1}{16.5}
$$

here the initial permeability, calculated from the measured static hysteresis loop data of the soft iron sample, was around $\mu_{i}=1000$.

Figure 4 depicts the calculated major loops.

In the second experiment the closed toroidal sample was magnetized into deeper saturation by applying a high $H_{m}=4125 \mathrm{~A} / \mathrm{m}$ maximum excitation. Following that, two $1.5 \mathrm{~mm}$ cut (effective $3 \mathrm{~mm}$ gap) were made in the toroid and the measurement was repeated.

This time however the maximum excitation was increased to approach $B_{m}=1.68 \mathrm{~T}$, achieved without the gap.

The loops were modelled by using the same numerical parameters at a larger $h_{m}$ maximum field excitation. The resulted loops (measured and calculated) are depicted in Figure 3. The agreement between the experimental result and the modelled one is extremely close.

Other additional factors like the internal, shape demagnetization factors and/or stresses were taken as negligibly small.

The separation and calculation of $\alpha_{k}$ and $n_{S}$ from the experimentally obtained $\alpha_{k} \cdot n_{S}$ product, requires the presence of two invariants in the transformation.

It is imperative to look at the two loops (un-sheared and sheared) at the same maximum magnetization (or as near as possible). Under this condition we can claim to have the necessary two invariants i.e. the coercivity and the maximum magnetisation.

It can be shown, that the two smaller processes (DR, DWAM) have often a negligible effect on the overall coercivity and experience shows that in most applications,

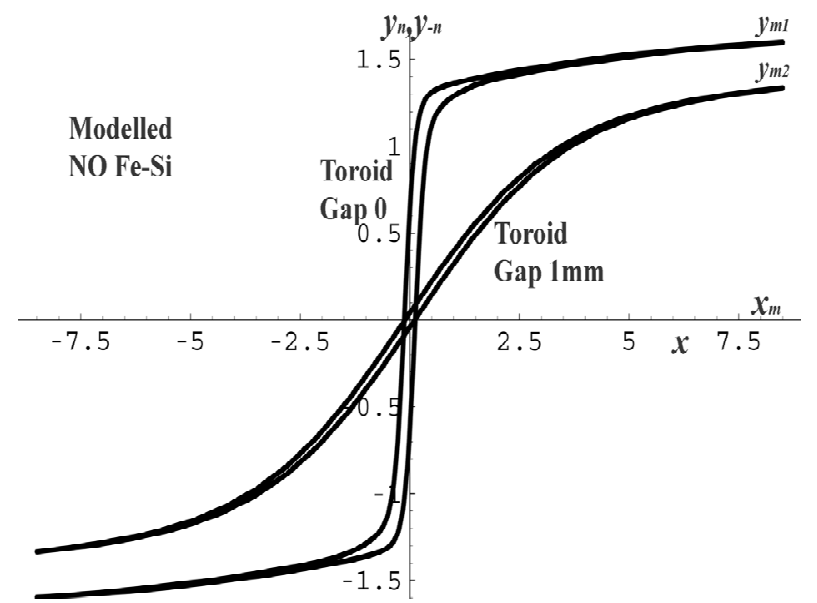

Figure 4. The modelled major loops before and after shearing. particularly for soft irons, it is accurate enough in most cases to use the equations describing the dominant process. The proposal is valid, however, when the DR and DWAM processes are not negligible. All resultant parameters, required for the transformation can be calculated (see Appendix) from the parameters of the processes involved. It is enough therefore to demonstrate the process by using one major component [22]. On this ground the present example is given entirely for illustrative purposes.

By using (2a), we can write for the sheared and the un-sheared dominant loop:

$$
a_{1} \tanh \alpha_{1}\left(h_{\mathrm{m} 1}-h_{c 1}\right)=a_{1} \tanh \alpha_{1} n_{S}\left(h_{m 1 s}-h_{c 1}\right)
$$

( $n_{S}$ being unity in the first case)

From this, the shearing coefficient $n_{S}$ may be calculated as:

$$
n_{S}=\frac{h_{\mathrm{m} 1}-h_{c 1}}{h_{\mathrm{m} 1 s}-h_{c 1}}
$$

where $h_{m 1}$ and $h_{m 1 s}$ are the amplitudes of field excitations necessary to achieve the same maximum magnetization in the un-sheared and the sheared sample respectively.

In soft steel, when the coercivity $h_{c}$ is small relative to the peak excitation, it is often good enough to use the ratio of the maximum field, from the saturation loop, measured by VSM. In the knowledge of $n_{S}$ the value of $\alpha_{\kappa}$ could be calculated from the measured $\alpha_{1} n_{S}$ product.

The external shearing coefficients, calculated from the measured data was $n_{s}=0.065$ for the first experiment (1 $\mathrm{mm}$ gap) and $n_{s}=0.02$ for the second one ( $3 \mathrm{~mm}$ gap). Some allowances should be made to the inherent internal demagnetization present in all type of measurement [2]. The estimated value of the internal demagnetization field was max $0.2 \mathrm{~T}$ [9].

From the second invariant, the ratio between the arctanh values of the remanences (sheared and un-sheared) (see Equation 2(b)) is also equal to $n_{S}$.

The measured remanence of the $1 \mathrm{~mm}$ gap sample was $0.032 \mathrm{~T}$ at $B_{m}=1.41 \mathrm{~T}$, while the corresponding modelled figure was $0.035 \mathrm{~T}$. In the case of the $3 \mathrm{~mm}$ gap sample, the measured and the modelled remanence figures were $0.0085 \mathrm{~T}$ and $0.0066 \mathrm{~T}$ respectively, taken at $B_{m}=1.5 \mathrm{~T}$. Based on these figures, $B_{r}$ the projected remanence of the loop, transformed back onto the unsheared $\left(n_{S}=1\right.$ and $\left.n_{D}=0\right)$ plane came to $0.636 \mathrm{~T}$.

It can be shown also, that the transformation does not change the area enclosed by the hysteresis loop, therefore sheared or un-seared, it has the same hyteretic losses.

\section{Conclusion}

It was proven, that shearing and/or un-shearing will not affect most of the static characteristic, material parame- 
ters of the soft sample. It is also shown, that the external demagnetization affects one parameter only, namely the effective slope (i.e. $n_{S} \alpha$ ) of the hysteresis loop. From the sample's geometry the shearing coefficient can often be calculated. With the proposed method and the knowledge of $N_{D}$ [2] one can exploit the advantage of the open geometry measuring methods for unusual samples (i.e. balls, sheets or rods with known $N_{D}$ ) and calculate the equivalent closed circuit static parameters.

The transformation is based on the two invariants, the coercivity and the maximum excitation.

This model independent transformation can be an useful tool in practice, in experimental work as well as in theoretical applications. The very close agreement between the theoretical and experimental results, the relative simplicity of the procedure and accuracy demonstrates the applicability of the proposed method in practical cases.

\section{REFERENCES}

[1] F. Fiorillo, "Measurement and Characterisation of Magnetic Materials," Academic Press, Torino, 2004.

[2] D. Jiles, "Introduction to Magnetism and Magnetic Materials," Chapman and Hall, New York, 1998.

[3] S. Foner, "The Vibrating Sample Magnetometer: Experiences of a Volunteer (Invited)," Journal of Applied Physics, Vol. 79, No. 8, 1996, p. 4740. doi:10.1063/1.361657

[4] D. B. Clarke, "Demagnetization Factors of Ringcores," IEEE Transactions on Magnetics, Vol. 35, No. 6, 1999, pp. 4440-4444. doi:10.1109/20.809135

[5] Zs. Szabo and A. Ivanyi, "Demagnetizing Field in Ferromagnetic Sheet," Physica B: Condensed Matter, Vol. 306, No. 1-4, 2010, pp. 172-177. doi:10.1016/S0921-4526(01)00999-1

[6] T. Nakata, N. Takahashi, K. Fujiwara, M. Nakano, Y. Ogura and K. Matshubara, "An Improved Method for Determining the DC Magnetization Curve Using a Ring Specimen," IEEE Transactions on Magnetics, Vol. 28, No. 5, 1992, pp. 2456-2458. doi:10.1109/20.179524

[7] J. Takacs, "Mathematics of Hysteretic Phenomena," Wiley-VCH, Berlin, 2003.

[8] J. Takács, "A Phenomenological Mathematical Model of Hysteresis," International Journal for Computation and Mathematics in Electrical and Electronic Engineering, Vol. 20. No. 4., 2001, pp. 1002-1005.

[9] E. Della Torre and F. Vajda, "Parameter Identification of the Complete-Moving-Hysteresis Model Using Major Loop Data," IEEE Transactions on Magnetics, Vol. 30, No. 6, 1994, pp. 4987-5000. doi:10.1109/20.334286
[10] D. C. Jiles and J. B. Thoelke, "Theory of Ferromagnetic Hysteresis: Determination of Model Parameters from Experimental Hysteresis Loops," IEEE Transactions on Magnetics, Vol. 25, No. 5, 1989, pp. 3928-3930. doi: $10.1109 / 20.42480$

[11] B. D. Cullity, "Introduction to Magnetic Materials," Addison-Wesley, Reading, 1972, p. 1014.

[12] D. X. Chen, J. A. Brug and R. B. Goldfarb, "Demagnetizing Factors for Cylinders," IEEE Transactions on Magnetics, Vol. 27, No. 4, 1991, pp. 3601-3619. doi:10.1109/20.102932

[13] J. H. Paterson, S. J. Cooke and A. D. R. Phelps, "Finite-Difference Calculation of Demagnetizing Factors for Shapes with Cylindrical Symmetry," Journal of Magnetism and Magnetic Materials, Vol. 177-181, 1998, pp. 1472-1473. doi:10.1016/S0304-8853(97)00788-9

[14] K. Tang, H. W. Zhang, Q. Y. Wen and Z. Y. Zhong, "Demagnetization Field of Ferromagnetic Equilateral Triangular Prisms," Physica B: Condensed Matter, Vol. 363, No. 1-4, 2005, pp. 96-101. doi:10.1016/j.physb.2005.03.007

[15] J. A. Osborn, "Demagnetizing Factors of the General Ellipsoid," Physical Review, Vol. 67, No. 11-12, 1945, pp. 351-357. doi:10.1103/PhysRev.67.351

[16] L. K. Varga, Gy. Kovács and J. Takács, "Modeling the Overlapping, Simultaneous Magnetization Processes in Ultrasoft Nanocrystalline Alloys," Journal of Magnetism and Magnetic Materials, Vol. 320, No. 3-4, 2008, pp. L26-L29. doi:10.1016/j.jmmm.2007.06.008

[17] J. Takacs, Gy. Kovacs and L. K. Varga, Journal of Magnetism and Magnetic Materials, Vol. 320, No. 20, 2008, p. 1016.

[18] J. Takacs, "Analytical Way to Model Magnetic Transients and Accommodation," Physica B: Condensed Matter, Vol. 387. No. 1-2, 2007, pp. 217-221. doi:10.1016/j.physb.2006.04.007

[19] J. Takacs and I. Meszaros, "Separation of Magnetic Phases in Alloys," Physica B: Condensed Matter, Vol. 403, No. 18, 2008, pp. 3137-3140. doi:10.1016/j.physb.2008.03.023

[20] J. Takacs, "The Everett Integral and Its Analytical Approximation," In: L. Malkinski, Ed., Magnetic Materials, Intech Publication, 2012.

[21] I. D. Mayergoyz, "Mathematical Models of Hysteresis and their Applications," Academic Press, Elsevier, New York, 2008.

[22] J. Takacs, Gy. Kovacs and L. K. Varga, "Hysteresis Reversal," Physica B: Condensed Matter, Vol. 403, No. 1316 , 2008, pp. 2293-2297. doi:10.1016/j.physb.2007.12.008 


\section{Appendix}

The single equivalent tanh function can be calculated from (14), (15), (16), and (17) as shown in (18), (19), (20) and (21).

$$
\begin{aligned}
y_{0 u}= & a_{0} \tanh \alpha_{0}\left(\xi_{u}-h_{c 0}\right)+f_{0} \\
= & a_{1} \tanh \alpha_{1}\left(h-h_{c 1}\right) \\
& +f_{01}+a_{2} \tanh \alpha_{2}\left(h-h_{c 2}\right)+f_{02}
\end{aligned}
$$

where the new integration constant is:

$$
\begin{gathered}
f_{0}=f_{01}+f_{02} \\
\alpha_{0}=\left(a_{1} \alpha_{1}\left(\operatorname{sech} \alpha_{1} h_{c 1}\right)^{2}+a_{2} \alpha_{2}\left(\operatorname{sech} \alpha_{2} h_{c 2}\right)^{2}\right) / a_{0}\left(\operatorname{sech} \alpha_{0} h_{c 0}\right)^{2}
\end{gathered}
$$

The remanence of the resultant hysteresis of the two process magnetization is described from (1b) and (2b) (for $h=0$ ) as:

$$
\begin{aligned}
a_{0} \tanh \alpha_{0} h_{c 0} & =a_{1} \tanh \alpha_{1} h_{c 1} \\
& +a_{2} \tanh \alpha_{2} h_{c 2}
\end{aligned}
$$

The maximum magnetization amplitude $a_{0}$ is:

$$
a_{0}=a_{1}+a_{2}
$$

From (15) $\alpha_{0} h_{c 0}$ can be calculated. From the firs de-

In the knowledge $a_{0}$ and $\alpha_{0}$ the $h_{c 0}$ can also be calculated.

$$
\xi_{u}=\left\{\operatorname{arctnh} \frac{1}{a_{0}}\left[a_{1} \tanh \alpha_{1}\left(h-h_{c 1}\right)+a_{2} \tanh \alpha_{2}\left(h-h_{c 2}\right)\right]+h_{c 0} \alpha_{0}\right\} / \alpha_{0}
$$

and

$$
\xi_{d}=\left\{\operatorname{arctnh} \frac{1}{a_{0}}\left[a_{1} \tanh \alpha_{1}\left(h+h_{c 1}\right)+a_{2} \tanh \alpha_{2}\left(h+h_{c 2}\right)\right]-h_{c 0} \alpha_{0}\right\} / \alpha_{0}
$$

From (15), (16), (17), (18) and (19) all parameters can be calculated for

$$
y_{0 u}=a_{0} \tanh \alpha_{0}\left(\xi_{u}(h)-h_{c 0}\right)+f_{0}
$$

and

$$
y_{0 d}=a_{0} \tanh \alpha_{0}\left(\xi_{u}(h)+h_{c 0}\right)-f_{0}
$$

as functions of the field excitation $h$.

This calculation can be repeated $\mathrm{n}-1$ times, for the equivalent set of parameters, where $n$ is the number of processes in magnetization. 